\title{
Timing in pigeons: The choose-short effect may result from pigeons' "confusion" between delay and intertrial intervals
}

\author{
LOU M. SHERBURNE \\ Wabash College, Crawfordsville, Indiana \\ and \\ THOMAS R. ZENTALL and DAREN H. KAISER \\ University of Kentucky, Lexington, Kentucky
}

\begin{abstract}
In conditional discriminations, when samples differ only in duration, pigeons typically show a choose-short effect (i.e., higher matching accuracy on short-duration-sample than on long-durationsample trials with increasing delay between sample and comparison stimuli). That this effect depends on the similarity of retention interval (RI) and intertrial interval (ITI) houselight illumination conditions has been taken as evidence that pigeons judge duration relative to a temporal background. In the present experiment, pigeons trained with duration samples and with the ITI either illuminated or not showed a choose-short bias only when the RI illumination on test trials was the same as the ITI illumination had been in training. The results support the hypothesis that the choose-short effect results from the pigeons' confusion between the ITI and the RI.
\end{abstract}

Pigeons can acquire a conditional discrimination (matching to sample) when the only conditional cue is the differential duration of an otherwise identical stimulus. A task of this type might involve, for example, reinforcement of a red-comparison choice after a 10 -sec white-keylight (long) sample and reinforcement of a green-comparison choice after a 2-sec white-keylight (short) sample. When trained on such a conditional discrimination, pigeons show divergent retention functions when delays are imposed between the samples and the comparison stimuli. That is, as the retention interval (RI) is increased, choice accuracy declines sharply on long-sample trials but remains high on short-sample trials.

This counterintuitive finding has been attributed to the subjective shortening of events over time (Spetch, 1987; Spetch \& Sinha, 1989; Spetch \& Wilkie, 1982, 1983). The longer it has been since an event occurred, the shorter its duration is judged to have been. Thus, as time passes since the presentation of a long sample, its duration is judged to be more similar to the reference memory for the short sample, and matching accuracy declines. As time passes since the presentation of a short sample, its duration is judged to be shorter as well. But subjective shortening of short samples does not lead to errors (i.e., it does

This research was supported by National Science Foundation Grant BNS-9019080 and National Institute of Mental Health Grant MH45979. Some of these data were presented at the 68th Annual Meeting of the Midwestern Psychological Association, Chicago, May 1996. The authors thank Janice Weaver, Brigette Cook, and Trisha Clement for their assistance with data collection. Correspondence should be addressed to L. M. Sherburne, Department of Psychology, Wabash College, Crawfordsville, IN 47933 (e-mail: sherburl@scholar.wabash.edu). not lead to choice of the comparison associated with long samples).

Spetch and Rusak (1992) recently suggested an alternative account of this choose-short effect. They proposed that the effect arises because pigeons judge the duration of a sample, on a trial-by-trial basis, relative to a temporal background composed of the intertrial interval (ITI) that precedes the sample and the RI that follows it. When the RI is increased, the sample duration is thus judged to be shorter, relative to the temporal background. Spetch and Rusak refer to this account as the relative duration hypothesis. This interpretation is supported by their finding that pigeons tested under RI conditions (houselight [HL] on or off) that match those of the ITI used in training show the choose-short effect but that pigeons tested under RI conditions different from the ITI condition used in training show parallel retention functions. They interpret the absence of the choose-short effect as disruption of temporal judgment caused by dissimilarity between the components of the temporal background. That is, when the ITI and RI are inconsistent, the pigeons become confused and fail to time samples appropriately.

But the fact that the choose-short effect depends on the similarity of the ITI and RI conditions suggests a different interpretation of the effect: It may result from confusion between ITIs and RIs. Pigeons trained with lit ITIs, for example, may "interpret" a lit RI followed by comparison choice as a no sample trial and choose short because no sample is judged to be more similar to a short sample than to a long sample (see Fetterman \& MacEwen, 1989; Kraemer, Mazmanian, \& Roberts, 1985). Similarly, pigeons trained with dark ITIs may interpret a dark RI followed by comparison choice as a no sample trial and 
choose short. According to this confusion account, the choose-short effect should depend, not on whether the RI condition is the same as the ITI condition on a given test trial but, rather, on whether the RI condition is the same as the ITI condition used in training.

In the present experiment, two groups of pigeons were trained on a 0-sec-delay conditional discrimination (comparisons were presented immediately upon offset of the sample) with long- and short-duration keylight samples. For Group Light, the HL was lit during the ITIs in training. For Group Dark, the HL was dark during the ITIs in training. All the birds were then tested with both lit and dark ITIs and lit and dark RIs. The procedure is illustrated in Table 1.

According to the confusion hypothesis, during 0-secdelay training, pigeons learn the meaning of the ITI. When, in test, the RIs are similar to the ITIs used in training, confusion results. Furthermore, according to the confusion hypothesis, the nature of the ITI on the current trial should not be important.

According to the relative duration hypothesis, however, the choose-short effect depends on the similarity of the ITI and the RI on the current test trial (i.e., the background against which the sample is presented). When the ITI and the RI on the current trial are not the same, the background is not uniform, and accuracy should be poor. Furthermore, according to the relative duration hypothesis, the relation between the ITI in training and the current RI should not be important.

Specifically, predictions based on the relative duration hypothesis and on the confusion hypothesis are the same for test trials preceded by an ITI that is the same as that used in training: The pigeons should show a choose-short effect when the ITI and delay conditions are the same (i.e., a lit ITI followed by a lit RI for Group Light; a dark ITI followed by a dark RI for Group Dark) and should not show divergent retention functions when the ITI and delay conditions are different (i.e., a lit ITI followed by a dark RI for Group Light; a dark ITI followed by a lit RI for Group Dark).

But predictions based on the two hypotheses are different for test trials preceded by an ITI that is different from that used in training. According to the relative duration hypothesis, the pigeons should show a chooseshort effect when the ITI and delay conditions are the same (i.e., a dark ITI followed by a dark RI for Group Light; a lit ITI followed by a lit RI for Group Dark) and show parallel retention functions when the ITI and delay conditions are different (i.e., a dark ITI followed by a lit RI for Group Light; a lit ITI followed by a dark RI for Group Dark). According to the confusion hypothesis, however, the pigeons should show precisely the opposite: parallel retention functions when the ITI and delay conditions are the same (i.e., a dark ITI followed by a dark RI for Group Light; a lit ITI followed by a lit RI for Group Dark) and a choose-short effect when the ITI and delay conditions are different (i.e., a dark ITI followed by a lit RI for Group Light; a lit ITI followed by a dark RI for Group Dark). Predictions based on the two hypotheses are contrasted in Figure 1.

\section{METHOD}

\section{Subjects}

The subjects were 6 experimentally naive White Carneau pigeons purchased from Palmetto Pigeon Plant (Sumter, SC). Purina Pro Grains, mostly provided during experimental sessions, maintained the pigeons at $80 \%-85 \%$ of their free-feeding weights. The birds were housed in individual wire mesh cages, with water and grit freely available. The colony was maintained under a 12:12-h light:dark cycle.

\section{Apparatus}

The experiment was conducted in a BRS/LVE (Laurel, MD) pigeon test chamber. The pigeon compartment in the sound-attenuated test chamber measured $31.5 \mathrm{~cm}$ high $\times 30 \mathrm{~cm}$ across the response panel $\times$ $30 \mathrm{~cm}$ from the response panel to the back wall. Three response keys $(2.5 \mathrm{~cm}$ square and $1.5 \mathrm{~cm}$ apart) were mounted in a horizontal row on the response panel. The bottom edge of the keys was $25 \mathrm{~cm}$ from the wire mesh floor. Behind each response key was a 12-stimulus inline projector (Industrial Electronics Engineering, Van Nuys, CA, with GE No. 1820 lamps). A white hue (no filter) could be presented on the center key. Red (R) and green $(\mathrm{G})$ hues (Kodak Wratten filters Nos. 26 and 60 , respectively) could be presented on the left and right keys. Access to a rear-mounted grain feeder could be obtained through a $5.1 \times$ $5.7 \mathrm{~cm}$ aperture, centered horizontally on the response panel and located midway between the floor and the response keys. Reinforcement consisted of timed access to Purina Pro Grains (mixed grain). A shielded $\mathrm{HL}$ mounted $7.6 \mathrm{~cm}$ above the center key provided general chamber illumination. White noise at $72 \mathrm{~dB}$ and an exhaust fan mounted on the outside of the chamber provided general sound masking. Experimental contingencies and data recording were controlled by a microcomputer located in an adjacent room.

\section{Procedure}

Pretraining. All the pigeons were trained to eat from the grain feeder and shaped, by the method of successive approximations, to peck a white hue on the center key and to peck R and G on the side keys. To encourage the pigeons to attend to the long- and short-duration samples that would be presented during training, reinforcement was provided for responding to the white hue on a fixed interval (FI) 10 -sec schedule on 12 of the 24 pretraining trials and on a FI 2 -sec schedule on the other 12 pre-

Table 1

Houselight Conditions During Training and Test

\begin{tabular}{|c|c|c|c|c|c|c|c|c|}
\hline \multirow[b]{3}{*}{ Group } & \multicolumn{3}{|c|}{ Training } & \multicolumn{5}{|c|}{ Test } \\
\hline & \multicolumn{3}{|c|}{ Houselight Conditions } & & \multicolumn{4}{|c|}{ Houselight Conditions for Both Groups } \\
\hline & ITI & Sample & Comparison Choice & Condition & ITI & Sample & Delay & Comparison Choice \\
\hline Light & Lit & Dark & Dark & Same & Lit & Dark & Lit & Dark \\
\hline \multirow[t]{3}{*}{ Dark } & Dark & Dark & Dark & Different & Lit & Dark & Dark & Dark \\
\hline & & & & Same & Dark & Dark & Dark & Dark \\
\hline & & & & Different & Dark & Dark & Lit & Dark \\
\hline
\end{tabular}




\section{Confusion Hypothesis Predictions}

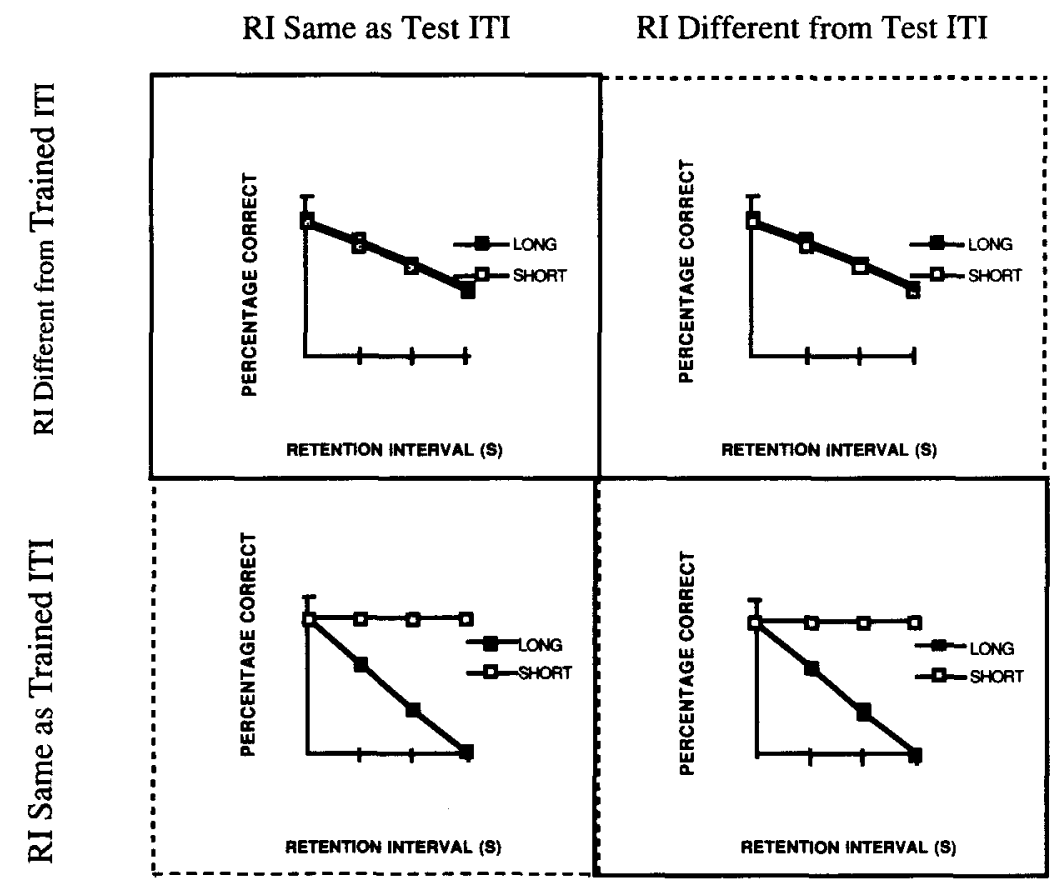

Relative Duration Hypothesis Predictions

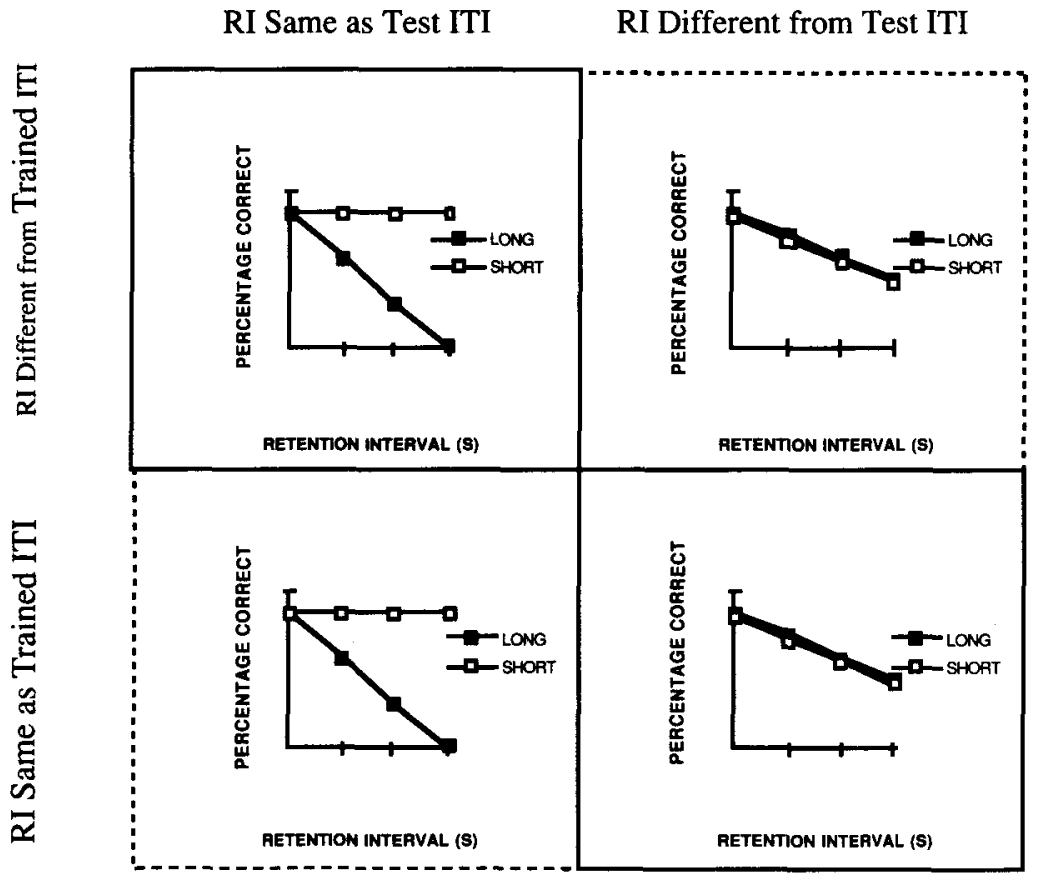

Figure 1. Differential predictions, based on the relative duration and confusion hypotheses, for delay performance by trial type (long vs. short samples). 


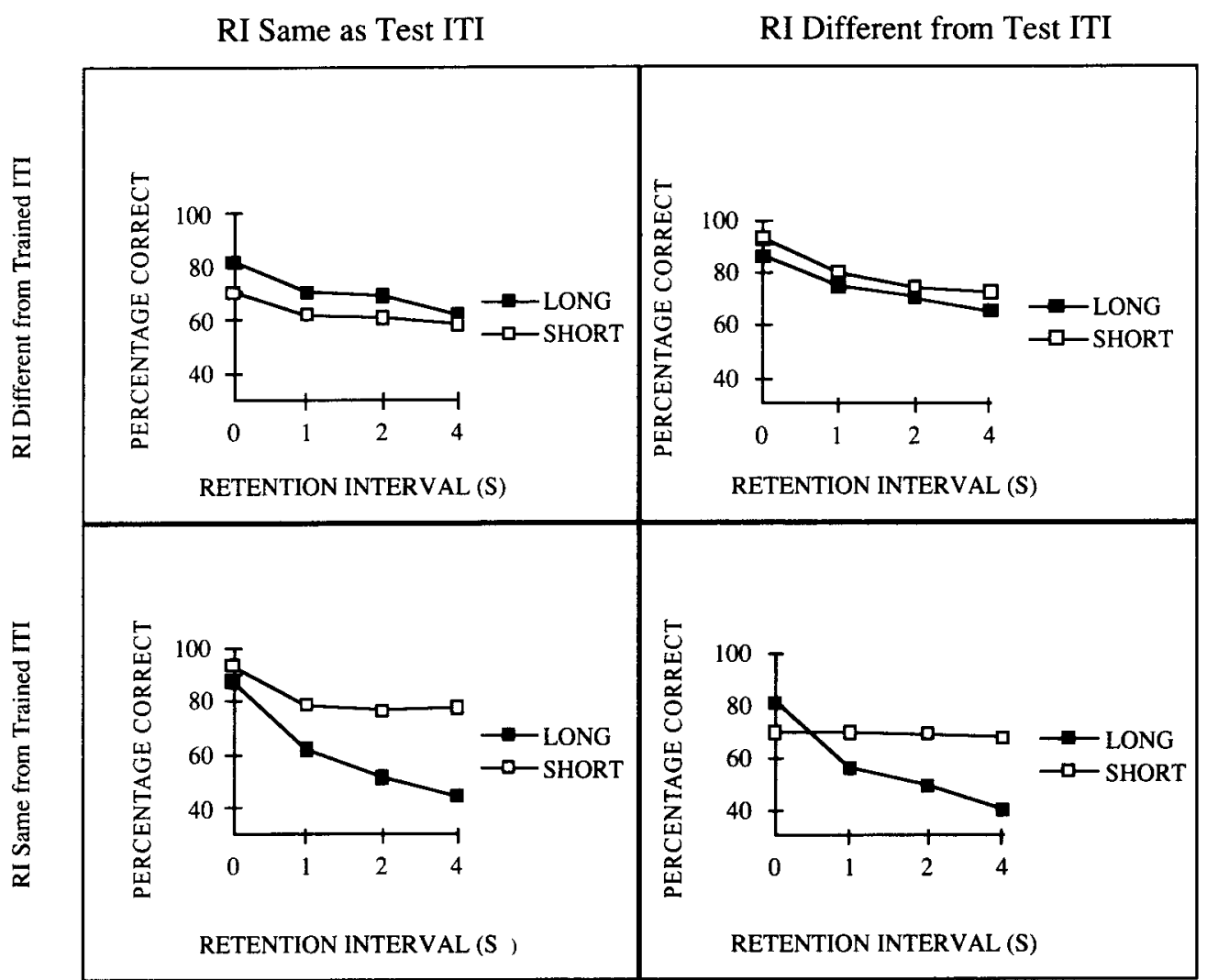

Figure 2. Matching accuracy for the two trial types (long vs. short samples) over 12 sessions of testing with $0-, 2-, 4-$, and 8-sec retention intervals between samples and comparisons. According to the relative duration hypothesis, the choose-short effect should occur when the intertrial interval (ITI) prior to a sample on a test trial matches the retention interval (RI) following the sample (bottom row) but not when they are different (top row). According to the confusion hypothesis, the choose-short effect should occur when the ITI in training matches the RI (left column) but not when they are different (right column).

training trials. The FI 10-sec and 2-sec presentations were balanced randomly within the session, with the restriction that neither schedule was in effect on more than 3 consecutive trials. The birds were then trained, during a single 24-trial session, to make a single peck to the $R$ and $G$ side key stimuli (fixed ratio [FR] 1). The stimulus presentations were balanced randomly within each session, with the restriction that neither hue nor presentation position (left or right) was repeated for more than 3 consecutive trials. Responses were reinforced with 3-sec access to mixed grain.

0-sec delay conditional discrimination training. Each trial began with either a long (L; fixed time [FT] $10 \mathrm{sec}$ ) or a short (S; FT $2 \mathrm{sec}$ ) presentation of the white hue on the center key. Following offset of the sample stimulus, $R$ and $G$ (the comparison stimuli) appeared on the side keys.

For 4 of the pigeons ( 2 from Group Dark and 2 from Group Light), a response to $R$ was reinforced following $L$ and a response to $G$ was reinforced following $\mathrm{S}$. For the other 2 pigeons, the sample-comparison associations were reversed (i.e., a response to $G$ was reinforced following $\mathrm{L}$, and a response to $\mathrm{R}$ was reinforced following $\mathrm{S}$ ). Reinforcement of a correct response consisted of a 1.5 -sec presentation of mixed grain, which was followed by a 10-sec ITI. An incorrect response was followed by the ITI alone. For the 3 pigeons in Group Light, the ITI was illuminated by the HL. For the 3 pigeons in Group Dark, the ITI was not illuminated. During each 96-trial session, the samples (48 L and $48 \mathrm{~S}$ ), correct comparisons ( $48 \mathrm{R}$ correct and $48 \mathrm{G}$ correct), and position of the comparison stimuli were balanced randomly within each session, with the restriction that none of these events was repeated on more than 3 consecutive trials. Two sequences of trial order were double-alternated over the sessions, which were conducted 6-7 days a week.

\section{Testing}

As each subject reached a criterion of 2 consecutive sessions at or above $90 \%$ correct, it began 128-trial sessions of testing with variable ITI illumination conditions ( $64 \mathrm{HL}$-on trials and $64 \mathrm{HL}$-off trials), variable RIs (32 0-sec RI trials, 32 2-sec RI trials, 32 4-sec RI trials, and 32 8-sec RI trials), and variable RI-HL-illumination conditions ( $64 \mathrm{HL}-\mathrm{on}$ trials and $64 \mathrm{HL}$-off trials; for purposes of comparison, half of the 0 -secdelay trials were randomly designated as $\mathrm{HL}$ on and the other half as HL off). The ITI HL-illumination conditions, samples, correct comparisons, $\mathrm{RI}, \mathrm{RI}-\mathrm{HL}$-illumination conditions, and comparison positions were balanced within each session in a factorial design. Each pigeon was tested for 12 sessions. As 0-sec (training) RI performance appeared to be quite disrupted by the delay testing (mean 0 -sec RI performance over the sessions $=69.0 \%$ ), each bird was retrained, following this testing, on the 0-sec-delay training task until the criterion performance level was regained. Each bird was then retested for 12 sessions as described above, except that the RIs were shortened to $0,1,2$, and $4 \mathrm{sec}$.

\section{RESULTS}

\section{Acquisition}

An analysis of variance (ANOVA) performed on the sessions-to-criterion data revealed no significant effect of group $[F(1,4)=.30, p=.61 ; M \mathrm{~s}=21$ and 23 sessions for Group Light and Group Dark, respectively]. 


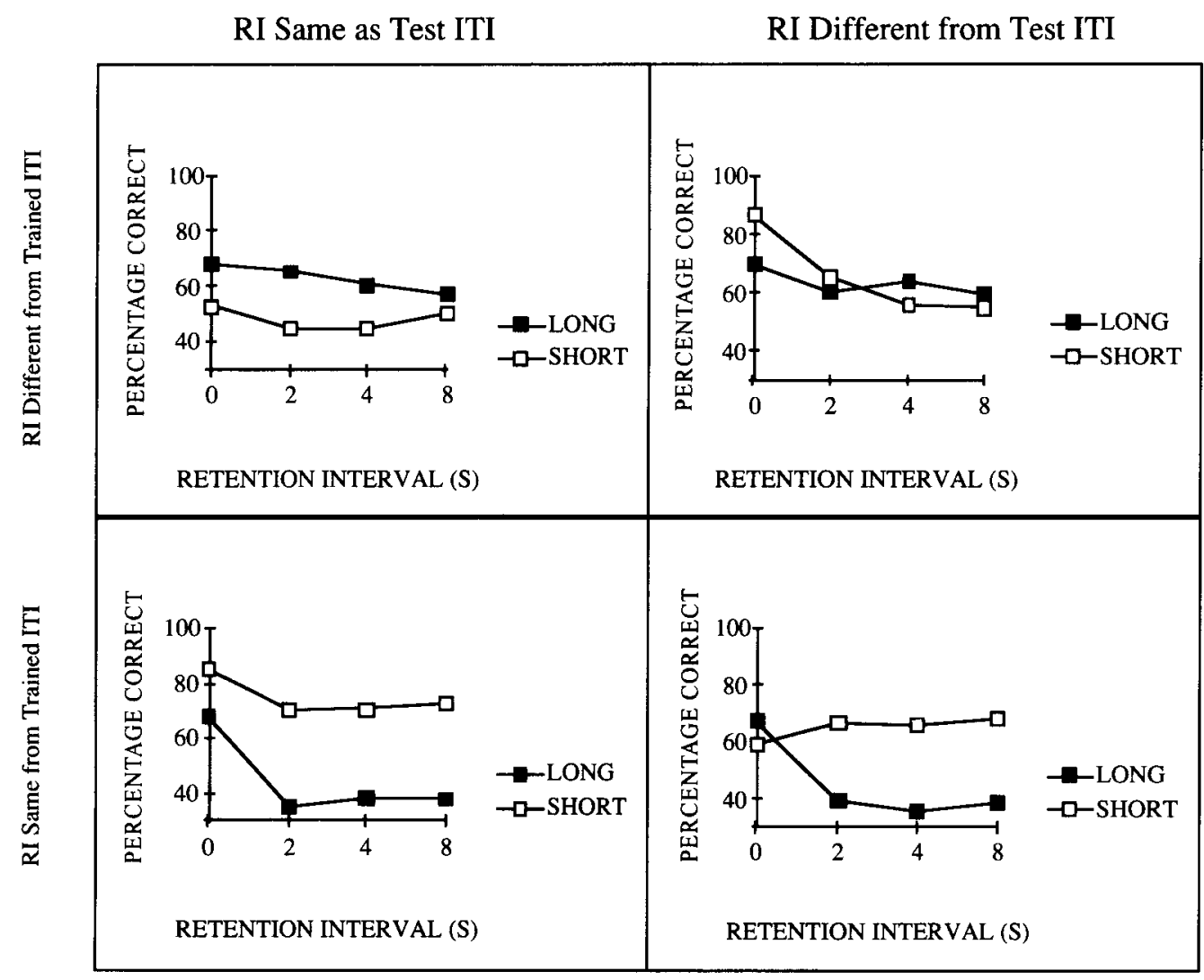

Figure 3. Matching accuracy for the two trial types (long vs. short samples) over 12 sessions of testing with 0-, 1-, 2-, and 4-sec retention intervals (RIs) between samples and comparisons. According to the relative duration hypothesis, the choose-short effect should occur when the intertrial interval (ITI) prior to a sample on a test trial matches the RI following the sample (bottom row) but not when they are different (top row). According to the confusion hypothesis, the choose-short effect should occur when the ITI in training matches the RI (left column) but not when they are different (right column).

\section{Test $1(0,2,4$, and 8 sec $)$}

The data from Test 1 appear in Figure 2. Each data point consists of 96 trials ( 8 trials/session pooled over the 12 test sessions). As can be seen in Figure 2, the pigeons showed parallel retention functions when the RIHL condition was different from the training-ITI condition, regardless of the test ITI-HL condition. However, the pigeons showed divergent retention functions when the RI-HL condition was the same as the ITI-HL condition. A five-factor mixed ANOVA was conducted on the data. The factors were group (trained with lit vs. dark ITIs), test ITI condition (lit vs. dark), sample type (L vs. S), RI condition (lit vs. dark), and RI duration ( $0 \mathrm{sec}$ vs. $2 \mathrm{sec}$ vs. $4 \mathrm{sec}$ vs. $8 \mathrm{sec})$.

The effect of most interest, from the point of view of the relative duration hypothesis, was that which reflected the effect of within-trial ITI/RI similarity (the two-way interaction of test ITI-HL condition and RI-HL condition) on the choose-short effect (defined by the within-panel two-way interaction: that between sample type and RI duration). The question here is, Does the choose-short effect depend on whether the RI-HL condition is the same as or different from the training ITI-HL condition on test trials? In other words, in Figure 2, are similar patterns of RI functions shown in the two panels on the left and in the two panels on the right, and are the patterns shown in the two left-hand panels different from those in the two right-hand panels? The analysis indicated that this was not the case: There was no significant four-way interaction of sample type $\times$ RI duration $\times$ test ITI condition $\times \mathrm{RI}$ condition $[F(3,12)=1.26, p=.33]$.

The effect of most interest, from the point of view of the confusion hypothesis, was that which reflected the effect of the similarity of the RI-HL condition to the training ITI-HL condition (the two-way interaction of group and RI-HL condition) on the choose-short effect. The question here is, Does the choose-short effect depend on whether the RI-HL condition is the same as or different from the training ITI-HL condition? In other words, in Figure 2, are similar RI functions shown in the two top panels and in the two bottom panels, and are the patterns shown in the top panels different from those in the two bottom panels? The answer was affirmative: The four-way interaction of sample type $\times$ RI duration $\times$ group $\times \mathrm{RI}-\mathrm{HL}$ condition was significant $[F(3,12)=$ $6.28, p=.008]$. When the HL condition during the RI 
matched the ITI-HL condition that had been used in training, a choose-short effect appeared, but not otherwise. And this effect was not influenced by the within-trial ITIHL condition: The five-way interaction was not significant $[F(3,12)=1.86, p=.19]$.

\section{Delay Test $2(0,1,2$, and $4 \mathrm{sec})$}

As mentioned earlier, 0 -sec test trial performance was quite low overall $(M=69.0 \%)$; thus, birds were retrained (all 0-sec-delay trials) and then retested with shorter (and, we hoped, less disruptive) RIs. Performance at the training RI was somewhat higher over the 12 sessions (mean 0 -sec RI performance $=82.6 \%$ correct) than in earlier testing, but the effects of interest were quite similar (see Figure 3). As with the results of Delay Test 1 , the four-way interaction of sample type $\times$ RI duration $\times$ group $\times \mathrm{RI}-\mathrm{HL}$ condition was significant $[F(3,12)=3.67, p=.04]$. And, again, neither the fourway interaction of sample type $\times$ RI duration $\times$ test ITI condition $\times \mathrm{RI}$ condition nor the five-way interaction was significant $[F(3,12)=1.31, p=.82$, and $F(3,12)=$ $1.85, p=.19$, respectively].

Two other effects are worthy of note. First, under conditions in which there is presumably no confusion (i.e., when RIs were different from the training ITI), one would expect better accuracy overall. Although this effect (the RI-HL condition $\times$ training ITI-HL condition) was not quite significant for the long-delay $(0,2,4$, and $8 \mathrm{sec})$ set $[F(1,4)=4.70, p=.10]$, it was significant for the shortdelay $(0,1,2$, and $4 \mathrm{sec})$ set $[F(1,4)=14.14, p=.02]$. Thus, matching accuracy improved when the presumed source of confusion on test trials was eliminated.

Second, there was a main effect of the RI-HL condition. Accuracy was significantly higher when the RIs were dark than when they were lit, both for the long-delay set $[F(1,4)=16.44, p=.02]$ and for the short-delay set $[F(1,4)=59.35, p=.002]$. This finding is consistent with results reported by others in which the HL has been lit during the RI (D'Amato \& O'Neill, 1971; Grant \& Roberts, 1976), and such effects have been interpreted in terms of retroactive interference from events occurring during the RI.

\section{DISCUSSION}

In this experiment, the pigeons in both training groups showed a choose-short bias that depended on whether the RI-HL condition matched the ITI-HL condition that was present in training (a result consistent with the confusion hypothesis). And the bias did not depend on whether the ITI-HL condition matched the ITI-HL condition on the current trial (a result which would be consistent with the relative duration hypothesis). Thus, when the meaning of the RI is clarified by making it highly discriminable from ITIs experienced during training, long and short samples appear to be remembered similarly to hue or shape samples (i.e., parallel retention functions result). But when the meaning of the Rl is obscured by its similarity to ITIs experienced during training, a choose-short effect results. These results suggest that the chooseshort effect arises from confusion between ITIs and RIs, rather than from judgment of duration relative to a temporal background.

It is not the case, however, that the choose-short effect is observed only in the performance of pigeons trained, as they were in the present study, with 0 -sec RIs. The effect is also observed in pigeons trained with longer RIs (e.g., 10 sec; see Spetch \& Rusak, 1989). A choose-short effect appears to result whenever the mean test RI is longer than that of training. The confusion account can be extended to account for such findings if one assumes that any lengthening of the RI (relative to that used in training) that makes the RI more temporally similar to the ITI used in training will result in some confusion. For example, pigeons trained with a 45-sec dark ITI and a 10-sec dark RI may confuse a I5or 20-sec dark test RI with the ITI.

If we assume that the choose-short effect results from an artifact associated with confusion of the ITI in training with the current RI, one would expect that matching accuracy on long-sample trials would be higher than that on short-sample trials. This outcome would be expected because long samples are presented for a longer duration and, thus, are likely to be more strongly represented in memory (see, e.g., Sacks, Kamil, \& Mack, 1972). According to this analysis, one might expect flatter or higher retention functions on long-sample trials than on shortsample trials.

Kraemer et al. (1985), too, suggested, that long-duration samples should be better encoded than short-duration samples. They found that with pigeons trained on a duration sample conditional discrimination with three response choices (long, short, and no sample) and a dark ITl, testing with dark RIs revealed a choose no sample effect rather than a choose-short effect--a result consistent with the confusion hypothesis (but see Kraemer et al., for an alternative interpretation). With increases in the RI, however, performance on long-sample trials dropped at a slower rate than did performance on short-sample trials. To explain this finding, Kraemer et al. argued that "the pigeon has longer to encode and rehearse the response instruction in the presence of the sample stimulus on long-sample trials than on short-sample trials" (p. 353).

Superior encoding of the long-duration sample may provide the basis for an explanation of a second nonparallel aspect of pigeons' memory for duration - one not addressed directly by the confusion account- the choose-long effect. After extensive training with a relatively long RI (of fixed duration), pigeons tested with shorter RIs showed higher accuracy on long-duration sample trials than on short-duration sample trials (Spetch, 1987). Testing with ITIs shorter than those used in training has also produced such a choose-long effect (Spetch \& Rusak, 1989). Spetch and Rusak proposed that the results of both the RI and the ITI manipulations can be explained in terms of the relative duration hypothesis, or greater intertrial proactive interference from the preceding trial (Overman \& Doty, 1980). Spetch and Rusak suggested that duration samples are represented analogically (the stronger their representation, the more likely it is that they will be judged to have been long) and that they lose strength over the passage of time since their presentation. According to Spetch and Rusak, the residual strength of the sample from the prior trial combines with the sample from the present trial through a process that they call temporal summation (Spetch \& Sinha, 1989). During training with a fixed RI, the pigeons learn to judge the strength of the current sample combined with the residual strength of prior samples. The effect of shortening the ITI would be to increase the residual strength of the samples from prior trials, thus resulting in a net increase in the strength of the sample representation at the time of comparison choice on all trials and producing a choose-long effect. This theory, based on analogical duration representations that weaken with time, can also account for the choose-long effect that results from shortening the RI from that experienced during training, because, on all trials, shortening the RI would increase the strength of the representation of the current sample at the time of comparison choice.

The confusion hypothesis (as originally stated) alone cannot account for the choose-long effect of the kind reported by Spetch and Rusak (1989). Some form of proactive interference appears to be needed to account for these effects, especially those involving manipulation of the ITI, but it is not clear that either analogical coding or temporal summation is necessary. First, as mentioned earlier, one can assume that long samples are better encoded than are short samples (there is more time to encode long samples). Second, one can assume that the strength of both the long- and short-sample codes will fade with time from their offset. During training with fixed delays, the animal learns to judge the strength of the weakened long-sample representation relative to the even weaker long-sample representations from prior trials. The same occurs for short-sample representations, but those representations are still weaker (because they were never as strong as the long representa- 
tions). Now, when either the ITI or the RI is shortened, it will increase the strength of prior long-sample representations. Under such conditions, representations of long samples from prior trials may be confused in strength with the reference memory of long-sample representations from training, and a choose-long effect may result.

If the choose-short effect that one finds when the introduced RIs are similar to the ITIs experienced during training results from confusion, one would think that the alleviation of that confusion (by making the RI distinctive from the ITI) should result in a higher level of accuracy. On the other hand, when the RI is distinctive from the ITI, the conditions present during the RI, at least initially, are novel. That is, for Group Light, the dark RIs represent a novel condition of total darkness, whereas for Group Dark, the RIs represent a novel condition of HL on. Since pigeons are known to be somewhat neophobic (see Zentall, Edwards, Moore, \& Hogan, 1981), the introduction of a distinctive, novel RI is likely to result, at least temporarily, in the disruption of task accuracy. In spite of this anticipated problem, task accuracy on trials with a novel (presumably nonconfusing) RI was actually higher than that on trials with a familiar (presumably confusing) RI. Although the difference in task accuracy on novel RI trials was not significantly different from that on familiar RI trials over the first 12 test sessions, the difference was significant over the second 12 test sessions. Thus, it is likely that the enhancement in task accuracy that resulted from the presentation of distinctive RIs was an underestimation of the magnitude of that effect.

The results of the present experiment suggest that pigeons do not judge duration relative to a temporal background. Furthermore, the results provide no support for the subjective-shortening hypothesis, because one would not expect that the passage of time would cause an interval to be subjectively shortened only if the RI matches the ITI. Although the present experiment identifies mechanisms that may underlie many of the effects that have been found when duration sample matching procedures have been used, it does not provide the means to determine how pigeons do judge duration.

The prospective-encoding view (Kraemer et al., 1985) accounts for divergent retention functions in terms of the similarity between a forgotten code after an RI and a short (peck R) code, but, as we have seen, confusion of the ITIs during training with the RIs may provide a better account of the typical choose-short effect. The prospective-encoding theory can also account for the choose-long effects reported by Spetch and Rusak (1989) by positing differential strengths of the prospective representations (prospective codes on long-sample trials are proposed to be stronger than those on short-sample trials) together with proactive interference from prior trials (as was discussed earlier).

A second alternative to the relative duration hypothesis is Killeen and Fetterman's (1988) behavioral-timing theory. According to this view, pigeons' comparison choices are cued by their behaviors at the time of choice (e.g., if involved in behavior A, peck R; if involved in behavior B, peck G). Again, to account for the choose-short effect reported under conditions of presumed confusion, the behavioral theory of timing includes the proposition that, if neither behavior is occurring at the time of comparison choice, a short response will be made (because short-sample behavior is more similar to not responding than is long-sample behavior). But, as with prospective-encoding theory, behavioral-timing theory is quite compatible with the notion of ITI/RI confusion and does not depend on the animal having such a choice bias. Furthermore, it is not difficult to imagine how behavioral-timing theory might account for Spetch and Rusak's (1989; Spetch, 1987) choose-long effects. More responding would be expected on long-sample trials than on short-sample trials, so those long-sample memories should be stronger. Furthermore, as described earlier, the same kind of proactive interference from prior trials may occur when shortening the ITI or the RI strengthens prior trial memories.

A more general implication of the present results is that we may need to consider more carefully the role of instructions in animal memory. It may be the case, for example, that the decrement in performance ob- served when animals are tested with RIs (regardless of RI/ITI similarity) is, to some extent, attributable to a training/test generalization decrement rather than to memorial processes. That is, the animal may accurately remember, for example, the sample that precedes a long RI, but its performance may suffer from a lack of adequate instructions. For example, pigeons trained to make response 1 after sample $A$ and to make response 2 after sample B may not know what to do after an RI, a novel event. It is not clear what testing procedures might be used to avoid a confounding of instructional failure and memory loss, but one might reserve strong conclusions about memorial processes to those experiments in which comparisons are made among conditions equally susceptible to instructional failure (e.g., across equally "confusing" RIs; see Zentall, 1997).

\section{REFERENCES}

D'AMaTO, M. R., \& O'NeILL, W. (1971). Effect of delay-interval illumination on matching behavior in the capuchin monkey. Journal of the Experimental Analysis of Behavior, 15, 327-333.

FetTERMAN, J. G., \& MACEwEN, D. (1989). Short-term memory for responses: The "choose-small" effect. Journal of the Experimental Analysis of Behavior, 52, 311-324.

GRANT, D. S., \& RoBERTs, W. A. (1976). Sources of retroactive inhibition in pigeon short-term memory. Journal of Experimental Psychology: Animal Behavior Processes, 2, 1-16.

KilleEn, P. R., \& FetTerman, J. G. (1988). A behavioral theory of timing. Psychological Review, 95, 274-295.

Kraemer, P. J., Mazmanian, D., \& Roberts, W. A. (1985). The choose-short effect in pigeon memory for stimulus duration: Subjective shortening versus coding models. Animal Learning \& Behavior, $13,349-354$

OVERMan, W. H., JR., \& Doty, R. W. (1980). Prolonged visual memory in macaques and man. Neuroscience, 5, 1925-1931.

SaCKS, R. A., Kamil, A. C., \& MaCK, R. (1972). The effects of fixedratio sample requirements on matching to sample in the pigeon. Psychonomic Science, 26, 291-293.

SPETCH, M. L. (1987). Systematic errors in pigeons' memory for event duration: Interaction between training and test delay. Animal Learning \& Behavior, 15, 1-5.

SPETCH, M. L., \& RuSaK, B. (1989). Pigeons' memory for event duration: Intertrial interval and delay effects. Animal Learning \& Behavior, 17, 147-156.

SPETCH, M. L., \& RuSAK, B. (1992). Time present and time past. In W. K. Honig \& J. G. Fetterman (Eds.), Cognitive aspects of stimulus control (pp. 47-67). Hillsdale, NJ: Erlbaum.

SPETCH, M. L., \& SinHA, S. S. (1989). Proactive effects in pigeons' memory for event duration: Evidence for analogical retention. Journal of Experimental Psychology: Animal Behavior Processes, 15, 347-357.

SPETCH, M. L., \& WILKIE, D. M. (1982). A systematic bias in pigeons' memory for food and light duration. Behaviour Analysis Letters, 2 , 267-274.

SPETCH, M. L., \& WILKIE, D. M. (1983). Subjective shortening: A model of pigeons' memory for event duration. Journal of Experimental Psychology: Animal Behavior Processes, 9, 14-30.

Zentall, T. R. (1997). Animal memory: The role of instructions. Learning \& Motivation, 7, 70-86.

Zentall, T. R., Edwards, C. A., Moore, B. S., \& Hogan, D. E. (1981). Identity: The basis for both matching and oddity learning in pigeons. Journal of Experimental Psychology: Animal Behavior Processes, 7, $70-86$.

(Manuscript received February 13, 1997; revision accepted for publication January 2, 1998.) 